OPEN ACCESS

Edited by:

Frank T. Robb,

University of Maryland, Baltimore,

USA

Reviewed by:

Amanda G. Oglesby-Sherrouse,

University of Maryland, Baltimore,

USA

Christopher John Grim,

United States Food and Drug

Administration, USA

Julia Van Kessel,

Indiana University Bloomington, USA

*Correspondence: Katherine García katherine.garcia@uautonoma.cl

Specialty section:

This article was submitted to

Evolutionary and Genomic

Microbiology,

a section of the journal

Frontiers in Microbiology

Received: 06 October 2016

Accepted: 22 December 2016

Published: 11 January 2017

Citation:

Pérez-Reytor D, Plaza N, Espejo RT, Navarrete P, Bastías $R$ and Garcia K (2017) Role of Non-coding Regulatory RNA in the Virulence of Human

Pathogenic Vibrios.

Front. Microbiol. 7:2160.

doi: 10.3389/fmicb.2016.02160

\section{Role of Non-coding Regulatory RNA in the Virulence of Human Pathogenic Vibrios}

\author{
Diliana Pérez-Reytor ${ }^{1}$, Nicolás Plaza ${ }^{1,2}$, Romilio T. Espejo ${ }^{2}$, Paola Navarrete$^{2}$, \\ Roberto Bastías $^{3}$ and Katherine Garcia ${ }^{1 *}$ \\ ' Centro de Investigación Biomédica, Facultad de Ciencias de la Salud, Instituto de Ciencias Biomédicas, Universidad \\ Autónoma de Chile, San Miguel, Chile, ${ }^{2}$ Institute of Nutrition and Food Technology, University of Chile, Santiago, Chile, \\ ${ }^{3}$ Laboratory of Microbiology, Institute of Biology, Pontificia Universidad Católica de Valparaíso, Valparaíso, Chile
}

In recent decades, the identification of small non-coding RNAs in bacteria has revealed an important regulatory mechanism of gene expression involved in the response to environmental signals and to the control of virulence. In the family Vibrionaceae, which includes several human and animal pathogens, small non-coding RNAs (sRNAs) are closely related to important processes including metabolism, quorum sensing, virulence, and fitness. Studies conducted in silico and experiments using microarrays and highthroughput RNA sequencing have led to the discovery of an unexpected number of sRNAs in Vibrios. The present review discusses the most relevant reports regarding the mechanisms of action of sRNAs and their implications in the virulence of the main human pathogens in the family Vibrionaceae: Vibrio parahaemolyticus, V. vulnificus and V. cholerae.

Keywords: sRNA, virulence, quorum sensing, pathogenic vibrios, Vibrio cholerae, Vibrio vulnificus, Vibrio parahaemolyticus, bioinformatics analysis

\section{INTRODUCTION}

The family Vibrionaceae is composed of a set of Gram-negative $\gamma$-proteobacteria present in marine environments (Heidelberg et al., 2002). The members of this family are easily isolated from different niches such as estuaries or deep water. They can also be found in planktonic form or on biotic and abiotic surfaces, establishing symbiotic or pathogenic interactions (Sun et al., 2013). The family includes important pathogens in aquaculture, such as Vibrio anguillarum, $V$. salmonicida, and V. harveyi (Thompson et al., 2004; Zhang et al., 2012). However, the most clinically significant members of the genus Vibrio are the human pathogens $V$. cholerae, V. vulnificus and V. parahaemolyticus (Thompson et al., 2004).

Most studies concerning the family Vibrionaceae have focused on $V$. cholerae, the species responsible for several outbreaks of cholera in places such as Bangladesh, Kolkata, and more recently Haiti (Antonova and Hammer, 2015). Today it is known that this microorganism produces a series of virulence factors that facilitate its colonization in the intestine, allowing a subsequent illness characterized by watery diarrhea, often fatal if untreated (Bradley et al., 2011; Cheng et al., 2015). The main virulence factors are the cholera toxin (CT) and the toxin co-regulated pilus (TCP; Bradley et al., 2011; Sengupta et al., 2016).

A second pathogen, V. vulnificus, is closely related to $V$. cholerae and is considered an opportunist pathogen that can cause septicemia in humans, frequently with fatal results (Alice et al., 2008). Infections produced by this pathogen represent $95 \%$ of all deaths related to the 
consumption of seafood in the United States, with a mortality rate close to $50 \%$. As a result, it is considered the most lethal food-transmitted pathogen in that country, and possibly in the world (Oliver, 2015). The main factor for its virulence is the presence of an antiphagocytic capsule (Thompson et al., 2004; Oliver, 2015). Finally, a third bacterial pathogen associated with the consumption of partially cooked or raw seafood is $V$. parahaemolyticus (Raghunath, 2015). This species is composed of numerous strains widely distributed in marine environments throughout the world (García et al., 2009, 2013; Loyola et al., 2015) but only a few cause diarrhea in humans. Virulent $V$. parahaemolyticus strains have different virulence factors, including adhesins, thermostable direct hemolysin (TDH) and TDH related hemolysin (TRH), as well as two different type-III (T3SS1 and T3SS2) and type-VI (T6SS1 and T6SS2) secretion systems, one in each of its two chromosomes (Letchumanan et al., 2014).

The infection process of these pathogenic bacteria requires the regulation of an arsenal of virulence factors that allow the bacteria to invade and survive inside their hosts, where they must acquire nutrients and resist environmental tension generated by the defenses of the host (Porcheron and Dozois, 2015). Recently, a new form of genetic regulation in bacteria has been revealed with the identification of small non-coding RNAs (sRNAs; Bardill and Hammer, 2012; Michaux et al., 2014). These RNAs form a heterogeneous group with 50 to 500 nucleotides (nt) that act via different mechanisms to modulate a wide range of cell responses (Repoila and Darfeuille, 2009; Silveira et al., 2010; Song et al., 2014), including regulation of virulence genes (Papenfort and Vogel, 2014). The regulation performed by these molecules can occur at all levels of gene expression, from the initiation of transcription, through translation control, to protein activity (Waters and Storz, 2009; Papenfort and Vogel, 2014). This control is achieved through a variety of mechanisms, including changes in RNA conformation, protein binding and RNA-RNA or RNA-DNA base-pairing (Waters and Storz, 2009; Oliva et al., 2015). Recent studies by massive RNA sequencing (RNA-seq) have revealed the wide distribution and large number of sRNAs present in bacterial genomes. But there is still a long way to go to understand the function of these molecules in the different networks including virulence regulation (Papenfort and Vogel, 2010; Sorek and Cossart, 2010).

The global prevalence of pathogens against humans and animals within Vibrio strengthens the need to understand how sRNAs participate in the regulation of different virulence factors associated with these bacteria and their effects on hosts (Letchumanan et al., 2014). This review discusses the most relevant reports regarding the mechanisms of action of sRNAs and their implications in the virulence of the main human pathogens in the family Vibrionaceae: V. parahaemolyticus, $V$. vulnificus and $V$. cholerae.

\section{CLASSIFICATION OF SRNAs}

Most sRNAs described to date can suppress certain cellular processes, inhibiting translation and decreasing the expression of the target gene, or acting on both processes simultaneously. However, it has also been documented that sRNAs can activate gene expression (Papenfort and Vanderpool, 2015). The main regulation mechanisms used by sRNAs described to date can be classified into five categories, as follows:

\section{sRNAs that Modulate Protein Activity}

Small non-coding RNAs that modulate protein activity are nonbase-pairing molecules and can interact directly with proteins to influence their activity by sequestration (Michaux et al., 2014). The system understood in this context is the global carbon storage regulator in the CsrA/RsmA family (Carbon storage regulator/Regulator of secondary metabolism; Lenz et al., 2005).

CsrA/RsmA proteins are global regulators of gene expression at a post-transcription level and play an important role in the expression of virulence factors in several proteobacterial pathogens (Babitzke and Romeo, 2007; Vakulskas et al., 2015). CsrA binds to mRNA, commonly inhibiting translation by blocking the ribosome binding site (RBS). This leads to the rapid degradation of their target mRNA and is fundamental to the regulation of some specific virulence systems required for host infection (Vakulskas et al., 2015). The activity of CsrA is regulated by sRNAs of the CsrB family (100 to more than 400 in length). These sRNA remove this protein from the target mRNAs by binding to different sites (Romeo et al., 2013). These sRNAs indirectly activate genes suppressed by CsrA, antagonizing the activity of this protein, and can be found in multiple copies per genome, performing redundant functions (Papenfort and Vanderpool, 2015).

\section{sRNAs that Are Transcribed Away from Their Targets (Coded in trans)}

Small non-coding RNAs that act in trans (sRNA-trans) are between 50 and $300 \mathrm{nt}$ in length; they are imperfectly complementary to one or more regions of the target mRNA, which is coded in another region of the genome. sRNA-trans participate in the response of the cell to changes in environmental conditions and are also involved in the regulation of virulence in pathogenic species (Malecka et al., 2015). The general mechanism of sRNA-trans is to occupy the RBS of a target mRNA by base pairing with the Shine-Dalgarno (SD) sequence or the start codon, although they can also interact with the mRNA coding sequence (Feng et al., 2015). As they are only partially complementary with their target, sRNA-trans generally require a chaperone, Hfq, to facilitate RNA-RNA interactions due to limited complementarity between the sRNA-target mRNA and to strengthen its regulation (Wagner, 2013; Van Puyvelde et al., 2015). Hfq belongs to the group of Sm-like (LSm) proteins which are also found in eukaryotes and archaea (Vogel and Luisi, 2011; Papenfort and Vanderpool, 2015). It may actively remodel the RNAs to melt inhibitory secondary structures and also may serve passively as a platform to allow sRNAs and mRNAs to sample potential complementarity (Waters and Storz, 2009). Besides, $\mathrm{Hfq}$ is frequently required to protect the sRNAs from cellular ribonucleases such as RNase E (Papenfort and Vanderpool, 2015), and for many intracellular and extracellular pathogens it is 
known that Hfq deficiency dramatically affects virulence and fitness (Escherichia coli, Kulesus et al., 2008; V. alginolyticus, Deng et al., 2016; Klebsiella pneumoniae, Chiang et al., 2011; Pseudomonas aeruginosa, Sonnleitner et al., 2003; Bordetella Pertussis, Bibova et al., 2013).

In case of sRNA-trans, a single mRNA can be regulated by more than one sRNA (Bardill and Hammer, 2012; Duval et al., 2014; Chang et al., 2015). This type of sRNA has been more widely studied and is the most abundant type of sRNA discovered to date. An example of this group are the sRNAs called Qrr (Quorum Sensing Regulatory RNA) that are involved in the regulation of bacterial quorum sensing (QS; Silveira et al., 2010). These Qrr sRNAs regulate target mRNAs either positively or negatively affecting translation, stability and/or processing of the target mRNA (Bardill and Hammer, 2012; Feng et al., 2015; Van Puyvelde et al., 2015). In the case of negative regulation, the sRNA-trans is paired close to the RBS of the target mRNA, blocking translation. In many cases this generates recruitment of RNase E and subsequent degradation of the mRNA. In positive regulation, the coded sRNA-trans performs an anti-antisense base-pairing in the $5^{\prime} \mathrm{mRNA}$ region. The ribosome binding site is thus revealed, promoting the stabilization of the target mRNA and hence translation. In this case, Hfq binds with both sRNA-trans and its corresponding mRNA target mediates the interaction (Fröhlich and Vogel, 2009; Feng et al., 2015). This can decrease the constant of apparent dissociation between the sRNA and its target mRNA and stabilize the sRNAs, protecting them from degradation by the RNase E (Bardill and Hammer, 2012; Duval et al., 2014).

\section{5' UTR Elements}

The regulator elements of $5^{\prime} \mathrm{UTR}$ (untranslated regions) are sequences that act in cis in the mRNA and have gained special attention in recent years. This group of elements is associated with the detection of a wide range of signals, including temperature (so-called thermoswitches; Nguyen and Jacq, 2014) and riboswitches (Smith and Strobel, 2011). The latter control gene expression by direct binding to ions or small molecules such as metabolites, co-enzymes, amino acids and nucleotide bases, causing changes in the secondary structure of the transcript (Cressina et al., 2011; Furukawa et al., 2012).

\section{sRNAs Coded in the Opposite Chain of the Regulated RNA (Coded in cis)}

Small non-coding RNA-cis are complementary to their target mRNA, and thus can interact autonomously. Their size also varies from approximately 100 to more than $300 \mathrm{nt}$, and may overlap with the $5^{\prime}$ or $3^{\prime}$ ends or be in a middle zone of the gene coded opposite the sRNA (Chang et al., 2015). These sRNAs are located in the antisense strand of their target RNA regions and their mechanism of action leads to the post-transcriptional downregulation of the target gene through perfect extended duplex (Oliva et al., 2015).

In terms of their mechanisms of action, it has been proposed that sRNAs-cis may have several advantages over their homologs that act in trans. Full and lengthy complementarity between the
sRNA and its target, observed in sRNA-cis, can lead to very stable duplexes, allowing regulation that is independent of proteins such as Hfq (Chang et al., 2015). Also, the cis position has the effect that sRNA and target are transcribed in close proximity, which can facilitate and improve interactions between the regulator molecules and their objective (Georg and Hess, 2011; Chang et al., 2015).

\section{The CRISPR/Cas System (Clustered Regularly Interspaced Short Palindromic Repeats)/CRISPR Associated}

CRISPR/Cas systems provide an adaptive immune mechanism in bacteria and archaea (Barrangou et al., 2007; Westra et al., 2014) which recognizes and degrades exogenous nucleic acid belonging to viruses and plasmids that invade the cell (Rath et al., 2015). The cleavage of foreign nucleic acid occurs through Cas proteins specifically guided by sRNA of approximately $30 \mathrm{nt}$ (Makarova et al., 2011). These guide sRNAs, called CRISPR RNAs (crRNAs), are found in a specific locus where each DNA sequence is flanked by a palindromic repeated sequence that participates in its genetic expression (Makarova et al., 2011; Rath et al., 2015). The immunological memory against future encounters with foreign nucleic acid is due to the incorporation of fragments of exogenous genetic material into the genome of the bacterium (Plagens et al., 2015; Rath et al., 2015). These systems are very diverse in terms of number, genetic structure and mechanism of action of Cas proteins (Makarova et al., 2011). Makarova et al. (2011) proposed a new classification of CRISPR/Cas systems of five types (types I-V) divided into two major classes. Class 1 systems have multi-subunit crRNA-effector complexes, whereas Class 2 system functions are carried out by a single subunit crRNA-effector module (Makarova et al., 2011).

\section{SRNAs INVOLVED IN THE VIRULENCE OF HUMAN PATHOGENIC Vibrio spp.}

It has been documented that sRNAs regulate several important processes in bacterial pathogens (Michaux et al., 2014; Sabharwal et al., 2015), including homeostasis of the outer membrane, QS, adaptation to stress, iron homeostasis, biofilm formation, virulence and host-cell contact, as well as development and metabolism (Liu et al., 2009; Papenfort and Vogel, 2014; Papenfort and Vanderpool, 2015; Sengupta et al., 2016). However, in Vibrios only few sRNAs have been studied experimentally and even fewer have been related to virulence.

Several sRNAs have been found inactive in cells lacking Hfq protein, which has been described as a master regulator of gene expression in bacteria, essentially due to its ability to mediate the interaction of sRNAs with their mRNA targets, including those related to virulence in Gram-negative bacteria (Kim et al., 2015; Feliciano et al., 2016). The association between Hfq and virulence in E. coli was revealed by Kulesus et al. (2008). Strains carrying a disruption in $h f q$ showed restricted motility, chemotaxis, ability to form biofilm and incapability to colonize effectively. The negative impact on all these processes reflects the capacity of this 
chaperone to interact with a wide range of different regulatory sRNA (Kulesus et al., 2008). Similarly, it has been reported that the deletion of the $h f q$ gene in Salmonella enterica serovar Typhimurium produces a non-motile strain which is highly attenuated in its ability to infect mice, invade epithelial cells, secrete virulence factors and to survive and proliferate within macrophages. These significant phenotypes suggest that $\mathrm{Hfq}$, as in E. coli, interacts with many sRNAs which are involved in virulence (Hébrard et al., 2012).

Hfq also appears to contribute in several distinct ways to the virulence of $V$. cholerae. It has been shown that $\Delta h f q$ strains fail to colonize the suckling mouse intestine (Ding et al., 2004), as well as deficient response to stress, changes in population behavior, altered metabolic regulation and loss of virulence (Shao and Bassler, 2012; Wagner, 2013). As Hfq often acts in conjunction with sRNAs, these molecules also play major roles in the control of cholera virulence (Ding et al., 2004).

One of these sRNA is VrrA (Vibrio regulatory $\underline{R} N A$ of OmpA; Sabharwal et al., 2015), whose expression is controlled by RpoE; it is capable of regulating the outer membrane porins OmpA and OmpT (Song et al., 2010; Sabharwal et al., 2015), the formation of outer membrane vesicles (OMV; Richard et al., 2010) and the expression of RbmC, which is important for biofilm formation (Sabharwal et al., 2015; Teschler et al., 2015). VrrA positively controls the release of OMV, causing an increase by repressing ompA (Song and Wai, 2009; Sabharwal et al., 2015). The formation and release of OMVs have gradually been more implicated in novel pathways of virulence factor delivery, since they have been proposed to play a role in several virulence mechanisms: periplasmic enzyme and transport, evasion of the immune system and toxin delivery. Additionally, attenuation in $V$. cholerae virulence was demonstrated using a deletion mutant of VrrA in an infant mouse model. Song et al. (2014) hypothesized that in the later stages of $V$. cholerae infection in the host, bacteria can move away from the epithelial surface into the intestinal lumen. During this time the bacteria may undergo a switch from attachment to the epithelial surface to detachment. This process may be associated with upregulation of VrrA, which can modulate expression of both a colonization factor (Tcp) and the attachment factor (RbmC; Song et al., 2014). VrrA mediates the downregulation of TcpA, a major virulence factor essential for host colonization in $V$. cholerae, possibly by binding directly to the $5^{\prime}$ UTR of $t c p A$ (Song and Wai, 2009). The regulation of $r b m C$ occurs by direct pairing with the $5^{\prime}$ end of the mRNA, inhibiting its translation and thus the formation of biofilms (Teschler et al., 2015). Notably, this regulation is not stringently dependent on Hfq protein, and hence VrrA represents the first example of direct regulation of sRNA on a biofilm matrix component, bypassing global master regulators (Song et al., 2014).

There are another two sRNAs, called TarA (ToxT activated RNA A; Richard et al., 2010) and TarB, which as their names imply are under the control of ToxT and involved in the regulation of virulence genes in V. cholerae (Bradley et al., 2011). Richard et al. (2010) observed that TarA, a highly conserved small regulatory RNA, unstable in the absence of Hfq and whose transcription is activated by ToxT, negatively regulates $p t s G$, a gene that codes the main glucose transporter in $V$. cholerae. The researchers showed that this sRNA influences glucose uptake by affecting the expression of PtsG though complementary bases between TarA and the $5^{\prime}$ region of the mRNA ptsG. However, the expression of TarA is activated by the virulence gene pathway in $V$. cholerae and not by glycolytic intermediates (Richard et al., 2010). These authors also showed that a classical V. cholerae O395 mutant lacking TarA is compromised for infant mouse colonization in competition with wild type, suggesting a role in the in vivo fitness of $V$. cholerae (Richard et al., 2010). However, regulation by TarA may not be equally critical in all $V$. cholerae strains since Bradley et al. (2011) did not observe difference in virulence comparing a tarA mutant strain with the wild type pandemic V. cholerae El Tor Biotype.

Also, it has been observed an increase in the mRNA tcpF in a $\operatorname{tar} B$ mutant, concluding that TarB negatively regulated the colonization factor TcpF and hence plays a negative role in virulence. Another study showed that TarB also decreases the expression of the transcription regulator VspR, which belongs to the Vibrio seventh pandemic island-1 (VSP-1; Bradley et al., 2011; Davies et al., 2012). VspR directly represses a gene that encodes a family of dinucleotide cyclases, which impacts virulence since the cyclic nucleotides play a role in biofilm formation, flagellum biosynthesis, DNA integrity and cell membrane stress. The signal molecule in $V$. cholerae seems to be predominantly cAMP-GMP, whose accumulation represses chemotaxis, and as an indirect effect enhances intestinal colonization by the bacterium (Davies et al., 2012).

Although most studies have been carried on $V$. cholerae, there are some studies that relate other Vibrios and regulation of virulence mediated by sRNA. In $V$. parahaemolyticus has been observed that the loss of Hfq provoked higher levels of $t d h$ expression (which codes TDH hemolysin; Nakano et al., 2008). Other genes involved in the virulence of $V$. parahaemolyticus, such as VP1680, vopC and vopt, which are effectors secreted by the T3SS were also overexpressed in $h f q$ mutant (Nakano et al., 2008; Nguyen and Jacq, 2014). These findings suggest that the expression of factors associated with the virulence of $V$. parahaemolyticus is negatively regulated by sRNAs, and Hfq may mediate the binding between these sRNAs and their target mRNA, thus affecting virulence.

Additionally in $V$. parahaemolyticus it has been shown that sRNA Spot 42 regulates the expression of the chaperone protein VP1682 in the T3SS1. Using electrophoretic mobility shift assay (EMSA), they proved that Spot 42 binds to the mRNA $v p 1682$ with the help of Hfq, and that the translation of this mRNA was inhibited in vitro in the presence of Hfq and Spot 42. In addition, in the deletion mutant of Spot $42(\Delta s p f)$, an increase was seen in VP1682 and VP1680 expression compared to the parent strain. Cytotoxicity assays in infected Caco-2 cell culture showed that the mutant $\Delta s p f$ has an increase in cytotoxicity compared to the wild type strain. These results show that Spot 42 regulates the expression of VP1682 post-transcriptionally, suggesting that it could contribute to the cytotoxicity of $V$. parahaemolyticus in vivo and constitute another example of the role of $\mathrm{Hfq}$ in the pathogenicity of these bacterial species (Tanabe et al., 2015).

The CRISPR/Cas system is another non-coding sRNA that plays an important role in virulence regulation. Sun et al. (2015) 
showed an association between the CRISPR sequence of 208 strains of $V$. parahaemolyticus and classical virulence factors of the species ( $t d h$ and $t r h$ ). Of $153 t d h$-positive strains, 149 isolates were identified as CRISPR positive, while $55 t d h$-negative isolates were identified as CRISPR negative, suggesting a close connection between these components. These authors mentioned significant association of CRISPR with the $t d h$ gene (Sun et al., 2013), suggesting an interesting role of sRNA in the evolution of pathogenesis. Li et al. (2014) analyzed the presence of CRISPR in 15 strains of $V$. parahaemolyticus of clinical and environmental origin. Using the CRISPR finder tool, the researchers found a total of six CRISPR elements. The results showed that environmental strains have fewer types of CRISPR than clinical strains, for which at least two CRISPR systems were found in each (Li et al., 2014).

\section{SRNAS IMPLICATED IN PROCESSES RELATED TO VIRULENCE IN HUMAN PATHOGENIC Vibrio spp.}

\section{Quorum Sensing}

Small non-coding RNAs can modulate the expression of genes associated with survival and virulence by pairing with untranslated regions of genes in response to cell density, through the QS pathway (Wen et al., 2016). QS is a process of cell communication by which a bacterial population expresses a set of genes in a coordinated way in response to the cell density in the population (Hammer and Bassler, 2007; Shao and Bassler, 2012, 2014; Van Kessel et al., 2015) through the production, release, detection and response to extracellular signal molecules called autoinducers (Tu and Bassler, 2007; Rutherford et al., 2011).

QS systems are widely distributed in Vibrio species (Zhang et al., 2012); the Qrr sRNA regulate multiple mRNA targets that code for QS regulatory components (Feng et al., 2015) through base-pairing with target mRNAs (Lenz et al., 2004; Svenningsen et al., 2008; Shao et al., 2013). These Qrr sRNAs appear in more than one copy per genome (Papenfort and Vanderpool, 2015). Qrr sRNAs activate the translation of AphA, a key regulator of low cell density (LCD), and suppress the translation of LuxR ( $V$. harveyi)/ HapR ( $V$. cholerae)/ SmcR (V. vulnificus)/ LitR (V. fischeri)/ ValR (V. alginolyticus) and OpaR ( $V$. parahaemolyticus), which is the main regulator of high cell density (HCD; Rutherford et al., 2011; Shao et al., 2013; Van Kessel et al., 2013b). Qrrs are highly conserved at the nucleotide level among Vibrio species but can vary in number and mechanism. For example, the five Qrr (Qrr1-5) of V. harveyi are additive because all of them are required to maintain wild-typelike repression of its master transcriptional regulator. Conversely, the four Qrr (Qrr1-4) of V. cholerae sRNAs are functionally redundant because any of its Qrr is sufficient to repress its master transcriptional regulator (Svenningsen et al., 2008). V. vulnificus has Qrr1-5 sRNAs that function additively, not redundantly, to repress SmcR (Wen et al., 2016). The only Qrr described in $V$. fischeri represses expression of the master regulator LitR (Kimbrough and Stabb, 2016) while in V. parahaemolyticus just three Qrr (Qrr2-4) sRNAs are known (Zhang et al., 2012).
However, this does not imply that other Qrr could exist in these species. New Qrrs could be still discovered because of the large number of sRNA described each year.

Qrr sRNAs also give feedback to suppress genes that code one of the QS synthase-receptor pairs, LuxMN, and the gene that codes the LuxO transcription factor (Zhang et al., 2012; Feng et al., 2015). Phosphorylated LuxO activates the transcription of the Qrr sRNAs, which bind and destabilize the mRNA $h a p R / \operatorname{luxR} / \operatorname{smcR} / o p a R$ as a function of Hfq (Tsou et al., 2011). However, the role of Qrr sRNA is not limited to QS, since they also regulate genes outside the QS circuit post-transcriptionally (Feng et al., 2015).

Although $V$. harveyi is not a human pathogen, is the species for which the QS process has been best characterized and has been considered as a general model for other Vibrio species, including human pathogens. In $V$. harveyi has been shown that Qrr3 controls its target mRNA through four different mechanisms, showing that a single sRNA molecule can play an important role in the regulation of its different targets by different mechanisms within the same bacterium, highlighting the versatility of these regulatory systems. In fact, Qrr3 sRNA suppresses luxR by catalytic degradation and $L u x O$ by sequestering, in a process that does not lead to the degradation of Qrr. It also suppresses luxM by coupled degradation and activates aphA by revealing the ribosome binding site, while at the same time the sRNA is degraded (Feng et al., 2015). The activation of the translation of AphA (Rutherford et al., 2011; Van Kessel et al., 2013a) indirectly activates the transcription of virulence and biofilm genes, which promotes bacterial colonization and infection (Wang et al., 2014), so an important function of sRNAs in Vibrios is the link between QS and virulence (Carignan et al., 2016). Also, HapR/LuxR/SmcR/OpaR regulates the expression of genes involved in the synthesis and degradation of c-di-GMP reducing the levels of this second messenger that controls the switch between biofilm formation and motility and other fundamental bacterial behavior including fimbrial synthesis, T3SS and RNA modulation (Srivastava and Waters, 2012; Wang et al., 2014; Sengupta et al., 2016).

In V.cholerae, sRNAs are critical components of the QS system and they constitute the first described example of sRNAs that regulate the expression of virulence genes in this pathogen (Tsou et al., 2011). There are sRNAs that participate directly in the expression of ToxT or are in turn controlled by ToxT, which is the main regulatory protein that controls the transcription of the genes $c t x A B$ and $t c p A-F$, considered the main virulence factor genes of this pathogen (Carignan et al., 2016) (Figure 1). sRNAs that are members of the Qrr regulon indirectly control the production of the virulence factors TCP and CT through regulation of AphA and HapR (Bardill and Hammer, 2012; Shao and Bassler, 2014). HapR negatively regulates the expression of $a p h A$ and directly suppresses biofilm formation by binding to the promoter of the gene $v p s T$, which codes a regulator of the exopolysaccharide production cluster $v p s$, and indirectly through $a p h A$, since AphA is a positive regulator of VpsT (Teschler et al., 2015; Carignan et al., 2016) (Figure 1). HapR also suppresses the transcription of $v p s R$, which codes another transcription factor that also activates genes involved in biofilm formation and 


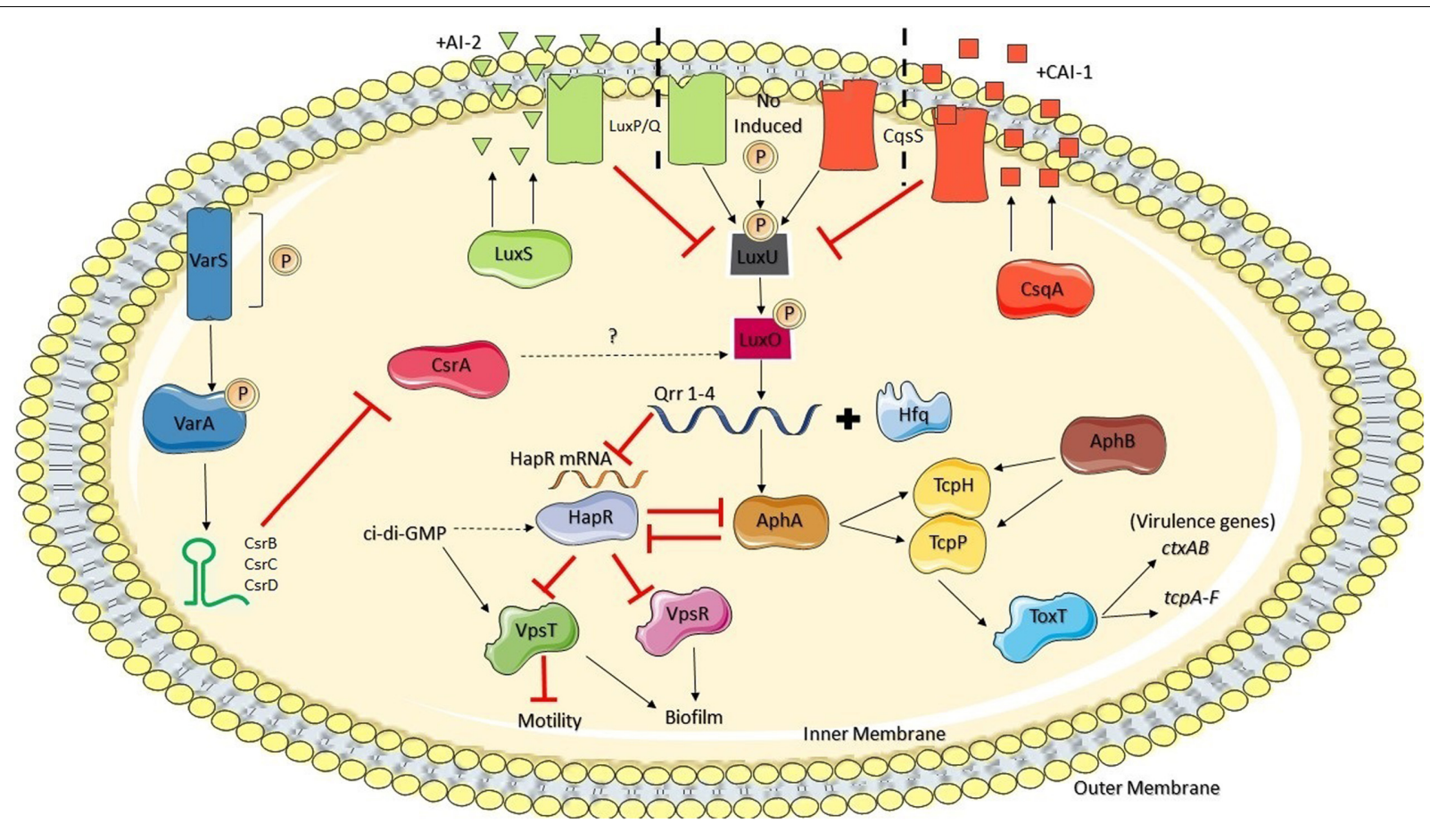

FIGURE 1 | Csr regulation circuit in V. cholerae and its relationship with HapR and Qrr sRNAs. (Adapted from Vakulskas et al., 2015, with permission of the publisher).

integrates signals from at least three different QS systems, which converge to the regulators of the LuxO response (Wang et al., 2014; Vakulskas et al., 2015) (Figure 1).

Notably, strains of $V$. cholerae modified to not produce Qrr sRNAs do not produce TCP or CT, and their capacity to colonize a lactating mouse model is severely lessened. HapR also positively regulates the transcription of hapA, a gene that codes the protease hemaglutinin (HA/protease), which is a virulence factor involved in the late stage of infection by $V$. cholerae, and the gene $\operatorname{prt} V$, which codes a metaloprotease that is both a virulence factor against the human host and a protection factor against predators in the environment (Lutz et al., 2013). This system is reinforced by a mutual suppression in which HapR suppresses the transcription of $a p h A$ and AphA suppresses the transcription of $h a p R$. Therefore, HCD induce the QS systems to reprogram gene expression, which stops the production of virulence factors and increases the production of transmission factors such as motility (Vakulskas et al., 2015).

Qrr sRNAs (Qrr1-4) act redundantly, and therefore the suppression of all four is required to avoid fully the repression of QS mediated by $\mathrm{Hfq}$ of genes downstream from HapR (Tsou et al., 2011). Moreover, in the absence of one or several Qrrs the others are overexpressed, compensating for the regulatory function. Svenningsen et al. (2008) observed that in $V$. cholerae HapR can activate the transcription of qrr genes, creating a negative feedback loop in the QS circuit. Therefore, HapR directly and indirectly suppresses its own production. Direct suppression of the promoter hapR by HapR only occurs at HCD, and it avoids overproduction of HapR at
LCD. On the other hand, post-transcriptional suppression of $h a p R$ by the Qrr sRNA feedback loop requires the presence of LuxO-P and HapR, and therefore it only occurs in the transition from HCD to LCD. The latter feedback loop drastically accelerates the transition of $V$. cholerae cells from the social mode to the individual cell mode (Svenningsen et al., 2008).

Another case is the sRNA VqmR, which was described by Papenfort et al. (2015) in $V$. cholerae through a differential RNA-seq. VqmR regulates biofilm formation by suppression of $v p s T$, which is controlled by QS through HapR, revealing that VqmR represents a previously unknown link between biofilm formation and QS. VqmR suppresses the expression of multiple mRNAs, including those that code for the toxin Rtx, and $v p s T$, which is required for biofilm formation, as demonstrated by the researchers through mutagenesis and microarray analysis (Papenfort et al., 2015). Shao and Bassler (2014) showed that LuxO functions through the sRNAs involved in QS to suppress T6SS, a new virulence factor in $V$. cholerae (Shao and Bassler, 2014; Cheng et al., 2015), using two mechanisms: the Qrr sRNAs suppress the production of T6SSactivator HapR, thus decreasing the expression; independently of HapR, the Qrr sRNAs suppress T6SS by direct basepairing with the mRNA that codes the long T6SS cluster. Regulation of T6SS Qrr sRNA is also conserved in pandemic and non-pandemic strains of $V$. cholerae (Shao and Bassler, 2014).

There is also a relation between virulence and QS in other human pathogen Vibrios. The expression of virulence factors 


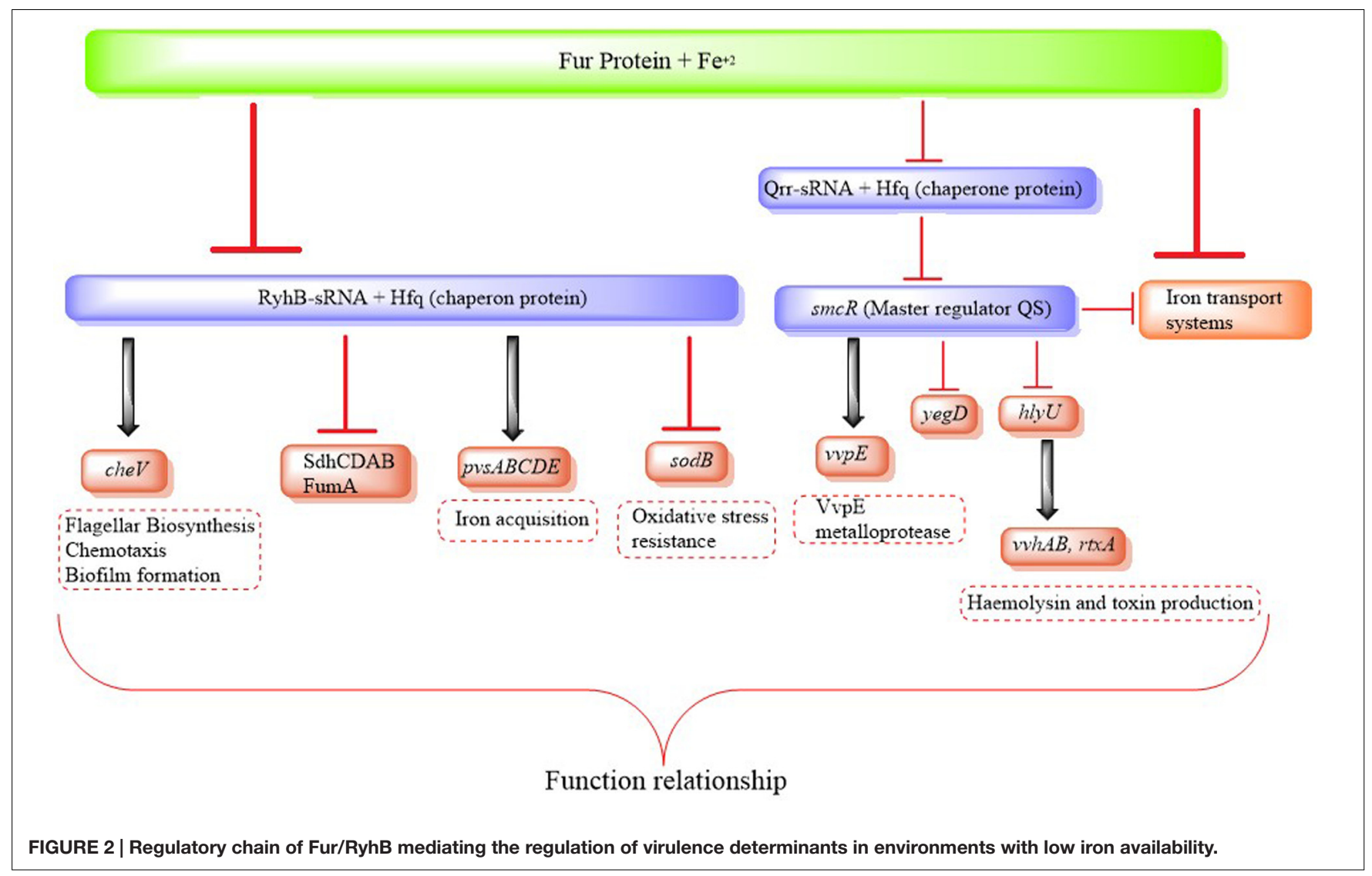

in $V$. parahaemolyticus is also modulated by QS, allowing differential genetic regulation under LCD and HCD conditions. OpaR controls opacity, biofilm formation and motility, represses T3SS1 regulons and oppositely, regulates the two T6SS in this bacterium (Gode-Potratz and McCarter, 2011; Burke et al., 2015). This QS-dependent regulation of T6SS is crucial as this system plays an important role in pandemic and nonpandemic strains of V. parahaemolyticus (Ceccarelli et al., 2013). Similarly, Qrr sRNAs affect the expression of pathogenicity genes in $V$. vulnificus by modulating the expression of the $s m c R$ (Figure 2). The quorum-sensing pathway is similar to $V$. cholerae, and $\mathrm{SmcR}$ is responsible for regulating the expression of diverse virulence factors (Wen et al., 2016). smcR mutants show attenuated cytotoxicity compared to the wild type (Lee et al., 2007). Wen et al. (2016) showed that Qrr sRNAs in $V$. vulnificus are regulated via LuxO and modulate the expression of virulence factors via SmcR. Interestingly, the authors also showed that Qrr sRNAs are responsive to iron concentration, and that iron and QS converge on Qrrs to control the expression of virulence factors (Wen et al., 2016) (Figure 2).

\section{The CsrA/RsmA Pathway and Its Implication on QS}

The CsrA/RsmA pathway has been identified in several $\gamma$-proteobacterial species, in which it regulates a wide range of cellular physiology: regulation of carbon metabolism, motility, biofilm formation, production of secondary metabolites and QS, thus affecting virulence (Babitzke and Romeo, 2007).

Maturation and dispersal of biofilms in $V$. cholerae, as well as CT and TCP production, are controlled by multiple systems independent of QS whose effects converge on a single regulator circuit (Rutherford and Bassler, 2012; Mey et al., 2015). CsrA influences the expression of virulence factors and QS through this circuit, connecting the response to autoinducers CAI-1 and AI-2 (Mey et al., 2015) (Figure 1). VarS/VarA (Virulence Associated Regulator) regulate QS indirectly through CsrA and three CsrA-inhibiting sRNAs: the aforementioned $\mathrm{CsrB}$ and also CsrC and CsrD (Lenz et al., 2005) (Figure 1). The response regulator VarA has been identified as a virulence regulator in this bacterium (Tsou et al., 2011). VarS/VarA has also been found to be involved in the control of HapR expression through LuxO and in the control of the CqsS and LuxPQ systems (Figure 1). Lenz et al. (2004) observed that $\mathrm{Hfq}$ is required for the regulation of QS due to the dependent chain of LuxO and that $h f q$ mutation affects the stability of the hapR messenger. Therefore, the VarS/VarA-CsrA/CsrBCD system converges with the QS system in $V$. cholerae to regulate the expression of the Qrr sRNAs and thus the entire QS regulon (Lenz et al., 2004). All these pathways converge to HapR, which thus represents a central node in this regulation network (Figure 1). 


\section{Iron Metabolism}

A fundamental element of homeostasis in microorganisms is iron, which is a cofactor of a large number of enzymes involved in essential biological processes (such as photosynthesis, respiration, DNA biosynthesis, etc.; Mey et al., 2005b; Repoila and Darfeuille, 2009; Porcheron and Dozois, 2015). However, excess free intracellular iron will give rise to reactions that generate toxic oxygen-reactive species (Nguyen and Jacq, 2014). Therefore internal iron levels must be rigorously controlled in accordance with physiological requirements, thus avoiding toxic effects.

Bacteria respond to fluctuations in iron concentration by coordinating the expression of genes that code for proteins involved in iron transport and storage, and genes that code for iron-dependent enzymes (Repoila and Darfeuille, 2009; Nguyen and Jacq, 2014). In Gram-negative bacteria, the main repressor of iron uptake systems is the ferric uptake regulation (Fur) protein. Fur complexed with $\mathrm{Fe}^{2+}$ binds to DNA (Bagg and Neilands, 1987), turning off the transcription of the iron uptake genes and limiting the entry of excess iron into the cell. When iron is limited in the cell, Fur is inactivated by the release of the iron cofactor, and the iron uptake genes are transcribed. Fur also regulates other genes involved in general metabolism and pathogenicity, thus the availability of iron in the host constitutes a key signal which coordinately regulates the expression of those genes (Tanabe et al., 2013).

Uncommonly, E. coli fur mutants have significantly reduced levels of total cellular iron despite the constitutive expression of iron uptake pathways (Abdul-Tehrani et al., 1999). This apparent paradox was explained by the presence of a sRNA, RyhB, which regulates the repression of numerous iron-containing proteins (Massé and Gottesman, 2002). In the absence of Fur, the RyhB sRNA have a key role in the control of iron homeostasis, repressing the synthesis of succinate dehydrogenase, aconitase, fumarase and iron superoxide dismutase. Total cellular iron is decreased as a result, despite a significant increase in cytosolic iron. Fur represses the synthesis of the RyhB, and RyhB in turn negatively regulates the synthesis of proteins that bind iron in the cell (Massé and Gottesman, 2002).

The mechanism of regulation by RyhB is post-transcriptional, requires $\mathrm{Hfq}$ and involves base pairing between RyhB and its mRNA target and subsequent RNase E-mediated degradation of the RyhB-mRNA duplex (Massé et al., 2003). Porcheron and Dozois (2015) distinguished three direct and indirect regulation mechanisms of virulence determinants by Fur and RyhB in response to environmental iron concentration. Fur and RyhB can act directly, regulating the expression of a gene, as is the case with genes involved in iron acquisition that are directly suppressed by Fur. The second mechanism involves other transcriptional regulators that determine invasion, acid resistance and toxin production; hence Fur/RyhB would indirectly regulate virulence. The last mechanism is related to the regulation of the locus of enterocyte effacement genes in E. coli, which occurs through the transcriptional regulator Ler; however, neither Fur nor RyhB directly regulates the expression of Ler (Porcheron and Dozois, 2015).
RyhB has a conserved region between $E$. coli and $V$. cholerae. The sRNA is longer in the latter, reaching lengths of $\sim 60 \mathrm{nt}$ on each side of the conserved region (Porcheron et al., 2014). Despite the conserved region, the two sRNAs differ in the degree and function of their regulons, since various mRNAs encoding ironcontaining proteins in E. coli are similarly regulated by RyhB in $V$. cholerae, but other transcripts regulated by RyhB in E. coli showed no RyhB-dependent regulation in $V$. cholerae and vice versa (Updegrove et al., 2015). Thus in both species RyhB has a core function for iron homeostasis, but in $V$. cholerae the sRNA has acquired new physiological roles (Updegrove et al., 2015).

RyhB is regulated by Fur in $V$. cholerae, and interacts with the protein $\mathrm{Hfq}$, required for the suppression of genes that code for the SodB superoxide dismutase and several enzymes in the tricarboxylic acid cycle, including succinate dehydrogenase SdhCDAB and fumarate reductase FumA (Figure 2). RyhB also modulates the expression of several genes that control motility, chemotaxis and biofilm formation in $V$. cholerae, since several genes involved in flagella biosynthesis and chemotaxis are negatively regulated in a $r y h B$ mutant of $V$. cholerae, and as mentioned above these are important processes in the virulence of the microorganism (Davis et al., 2005; Mey et al., 2005a) (Figure 2). Both Hfq and RyhB can be co-purified from $V$. cholerae and high levels of RyhB are only detectable in the presence of $\mathrm{Hfq}$, suggesting that as with E. coli, Hfq stabilizes this sRNA (Davis et al., 2005). Using microarray analysis, Mey et al. (2005b) showed that numerous genes that are involved in iron acquisition, including $f h u A C, f e o A B$ and $\operatorname{irg} A$, were increased when RyhB was expressed. In addition, a fur mutant of $V$. cholerae was unable to use pyruvate, succinate or fumarate as carbon sources (Litwin and Calderwood, 1994; Mey et al., 2005a). This suggests that $V$. cholerae may use a system analogous to the E. coli RyhB mechanism for regulating genes encoding iron-containing proteins and those involved in iron metabolism.

Homologs of $V$. cholerae RyhB have been identified in other Vibrio species including V. vulnificus and V. parahaemolyticus (Mey et al., 2005a; Porcheron et al., 2014). In V. vulnificus iron is necessary for growth and increased host mortality in vivo (Wen et al., 2016). Alice et al. (2008) carried out competitive experiments in an "iron-overloaded mouse" model with the wild type strain of $V$. vulnificus, which showed that the mutant $r y h B$ is less virulent. While the fur mutant (lethal dose) $\mathrm{LD}_{50}$ is the same as the wild type strain, the $\mathrm{LD}_{50}$ of the $\mathrm{RyhB}$ mutant is more than 30 times greater than that of the wild type strain (Alice et al., 2008; Porcheron and Dozois, 2015). The researchers attributed the attenuated virulent phenotype of the $V$. vulnificus $r y h B$ mutant to a defect in the growth observed in this mutant under limited iron conditions. However, in a double mutant $\Delta r y h B$ fur::pDM4 $\Delta$ lacZ growth was recovered under limited iron conditions, although the virulence remained attenuated, possibly implying that other genes related to the virulence of V. vulnificus may be directly or indirectly under the control of RyhB (Alice et al., 2008).

Vibrio parahaemolyticus can use several siderophores, including an analog of vibrioferrin, exogenous aerobactin, ferrichrome and enterobactin to acquire iron under low iron conditions (Tanabe et al., 2013). It has been observed that the 
production of vibrioferrin decreased in a $r y h B$ deletion mutant of $V$. parahaemolyticus compared to the parent wild type strain. This suggests that RyhB positively regulates the production of vibrioferrin, and the evidence indicates that this probably occurs through Hfq-dependent stabilization of the operon pvsOpm which is involved in its biosynthesis. This was the first report to state that RyhB positively regulated a polycistronic mRNA (Tanabe et al., 2013).

\section{BIOINFORMATICS ANALYSIS APPLIED TO THE STUDY OF SRNAS}

The continuous discovery of new sRNAs and their potential action mechanisms has increased the interest in identifying associations between sRNA and bacterial pathogenesis (Papenfort and Vogel, 2010). Computational predictions conducted with the enormous number of microbial genomes currently available and experimental tracing using new technologies such as microarrays and RNA-seq have discovered an unexpected number of RNA loci (Papenfort and Vogel, 2010; Pain et al., 2015). Silveira et al. (2010) used a combination of in silico searches validated by microarray analysis and RT-PCR to find and characterize a diverse range of sRNAs in the genomes of $V$. alginolyticus, $V$. campbellii, $V$. mimicus and $V$. communis. This approach culminated in the identification of 31-38 genes encoding putative sRNAs per species (Silveira et al., 2010).

TargetRNA is a bioinformatics tool designed to predict mRNA targeted by the action of sRNA in bacteria through base complementarity. It has been validated experimentally in E. coli using both northern blot and microarray experiments (Tjaden, 2008). The predictions of this program have also been corroborated in $V$. cholerae, in which several sRNAs have been identified using gene tracing and computational methods (Song and Wai, 2009). A more recent actualization of TargetRNA2 includes new characteristics such as conservation of sRNAs in other bacteria, secondary structure of the sRNAs, secondary structure of each possible target mRNA and the hybrid energy between the sRNA and the target mRNA (Kery et al., 2014). Sabharwal et al. (2015) performed an in silico analysis with TargetRNA to identify new VrrA-targeted mRNAs, finding vrp as a possible sRNA target. This prediction was complemented with another tool, RNAhybrid, to authenticate the pairing region between VrrA and $\operatorname{vrp}$ (Sabharwal et al., 2015). Another tool is the software package sRNAPredict. Davis and Waldor (2007) used this program to identify the sRNA MicX. This sRNA negatively regulates the expression of $v c 0972$, which encodes an uncharacterized OMP, and vc0620, which encodes the periplasmic component of a peptide $\mathrm{ABC}$ transporter in $V$. cholerae. This prediction was made based on the proximity of a potential rho-independent terminator of intergenic sequences conserved between $V$. cholerae, $V$. parahaemolyticus and V. vulnificus (Davis and Waldor, 2007). More recent tools to detect sRNA such as CopraRNA and IntaRNA were described by Wright et al. (2014). CopraRNA is able to use the whole genome available on platforms (NCBI, Rfam) to obtain three or more sequences of homologous sRNA for a set of given organisms. In contrast, IntaRNA can also be applied to non-whole genome screens using smaller sets of RNA molecules as input, in the absence of homologous sRNA. It is focused on the prediction of interactions between two RNA molecules and domain interaction. Both complementary results should generally be evaluated, because this allows characterizing the binding site region of the target (Wright et al., 2014).

The main challenge in the study of new sRNAs is not just their identification, but also elucidating their targets (Kery et al., 2014). sRNAs-trans normally have limited complementarity with their target mRNAs (Kery et al., 2014) and sRNAmRNA hybrids occur in relatively short regions that are not easily distinguishable from many other hybrids formed by random pairs of transcripts (Pain et al., 2015). Because of these limitations, predicting the regular function of sRNAs through bioinformatics identification of their target mRNAs has proven difficult (Song and Wai, 2009). Besides, the use of prediction programs presents a series of problems, as described by Pain et al. (2015), who evaluated sRNA target prediction programs using reliable sRNA/target pairs of $E$. coli. They noted that despite continuous improvements in computer programs, the number of true biological targets with low scores (false negatives) and the non-targets with high scores (false positives) remains counterproductive. This may be partly because many true targets have yet to be validated experimentally. sRNA-mRNA interaction in vivo can be positively affected by the presence of sRNA chaperones such as $\mathrm{Hf}$, which can significantly influence computer predictions. Another reason is that most programs predict possible targets based on free energy values of binding. However, high binding energy does not necessarily imply efficient processing of the target mRNA by the Hfq-sRNA complex (Pain et al., 2015).

\section{CONCLUSION}

Several studies regarding sRNAs in the full genomes of bacteria carried out in recent years have revealed that they are highly variable, widely distributed and of diverse functionality. It has also been shown that they play a crucial role in many biological processes such as development, metabolism, adaptation to stress, QS and virulence. The discovery of new sRNAs in the family Vibrionaceae, particularly in pathogenic species, and the knowledge of the targets they regulate is continuously increasing. Thus establishing a connection between the regulation mediated by sRNAs and virulence would shed new light on the understanding of the mechanisms of infection in these species. Despite the difficult task of identifying the targets regulated by sRNAs, the growing number of sRNAs identified together with their target genes in a wide range of bacteria has allowed comparisons between species (Updegrove et al., 2015). Although most studies in the Vibrionaceae have focused on V. cholerae, several laboratories are working on the implications of sRNAs in the virulence mechanisms of other human pathogenic species of this family. In this review we discussed some well characterized sRNAs, but there are many 
more sRNA described in databases whose function is unknown, and consequently much information remains to be elucidated. Currently most sRNAs in $V$. parahaemolyticus and $V$. vulnificus are described only by homology, thus experimental validation is highly required. Even though the bioinformatics tools are increasingly advanced, they are just an initial approximation in the study of sRNA and its targets, but the interaction between the mRNA-sRNA complex and the sRNA mechanisms of action can only be verified with experimental techniques, including EMSA, mutant development and RNA expression assays (such as microarrays and RNA-seq). Additionally, the fact that there are some contradictory effects in the function of one sRNA depending on the strain used calls for caution when these results are extrapolated to other species. Thus although there has been much progress in the study of sRNA in Vibrionaceae, more experimentation is needed to determine with certainty which sRNAs govern the virulence mechanism of human pathogenic Vibrio.

\section{REFERENCES}

Abdul-Tehrani, H., Hudson, A. J., Chang, Y. S., Timms, A. R., Hawkins, C., Williams, J. M., et al. (1999). Ferritin mutants of Escherichia coli are iron deficient and growth impaired, and fur mutants are iron deficient. J. Bacteriol. 181, 1415-1428.

Alice, A. F., Naka, H., and Crosa, J. H. (2008). Global gene expression as a function of the iron status of the bacterial cell: influence of differentially expressed genes in the virulence of the human pathogen Vibrio vulnificus. Infect. Immun. 76, 4019-4037. doi: 10.1128/IAI.00208-08

Antonova, E. S., and Hammer, B. K. (2015). Genetics of natural competence in Vibrio cholerae and other Vibrios. Microbiol. Spectr. 3, 1-18. doi: 10.1128/ microbiolspec.VE-0010-2014

Babitzke, P., and Romeo, T. (2007). CsrB sRNA family: sequestration of RNAbinding regulatory proteins. Curr. Opin. Microbiol. 10, 156-163. doi: 10.1016/j. mib.2007.03.007

Bagg, A., and Neilands, J. B. (1987). Molecular mechanism of regulation of siderophore-mediated iron assimilation. Microbiol. Rev. 51, 509-518.

Bardill, J. P., and Hammer, B. K. (2012). Non-coding sRNAs regulate virulence in the bacterial pathogen Vibrio cholerae. RNA Biol. 9, 392-401. doi: 10.4161/rna. 19975

Barrangou, R., Fremaux, C., Deveau, H., Richards, M., Boyaval, P., Moineau, S., et al. (2007). CRISPR provides acquired resistance against viruses in prokaryotes. Science 315, 1709-1712. doi: 10.1126/science.1138140

Bibova, I., Skopova, K., Masin, J., Cerny, O., Hot, D., Sebo, P., et al. (2013). The RNA chaperone $\mathrm{Hfq}$ is required for virulence of bordetella pertussis. Infect. Immun. 81, 4081-4090. doi: 10.1128/IAI.00345-13

Bradley, E. S., Bodi, K., Ismail, A. M., and Camilli, A. (2011). A genome-wide approach to discovery of small RNAs involved in regulation of virulence in Vibrio cholerae. PLoS Pathog. 7:e1002126. doi: 10.1371/journal.ppat. 1002126

Burke, A. K., Guthrie, L. T. C., Modise, T., Cormier, G., Jensen, R. V., McCarter, L. L., et al. (2015). OpaR controls a network of downstream transcription factors in Vibrio parahaemolyticus BB22OP. PLOS ONE 10:e0121863. doi: 10.1371/ journal.pone.0121863

Carignan, B. M., Brumfield, K. D., and Son, M. S. (2016). Single nucleotide polymorphisms in regulator-encoding genes have an additive effect on virulence gene expression in a Vibrio cholerae clinical isolate. mSphere 1, e253-e216. doi: $10.1128 / \mathrm{mSphere} .00253$-16.Editor

Ceccarelli, D., Hasan, N. A., Huq, A., and Colwell, R. R. (2013). Distribution and dynamics of epidemic and pandemic Vibrio parahaemolyticus virulence factors. Front. Cell. Infect. Microbiol. 3:97. doi: 10.3389/fcimb.2013.00097

Chang, H., Replogle, J. M., Vather, N., Tsao-Wu, M., Mistry, R., and Liu, J. M. (2015). A cis -regulatory antisense RNA represses translation in Vibrio cholerae

\section{AUTHOR CONTRIBUTIONS}

DP-R and KG conceived the idea and wrote the manuscript. NP made the figures. RE wrote the bioinformatics item. PN and RB wrote about CRISPR and Vibrio vulnificus items, respectively. All authors read and approved the final version.

\section{FUNDING}

The authors acknowledge Fondecyt project 11140257 and Fondecyt proyect 11140412, Chile.

\section{ACKNOWLEDGMENT}

We thank Oscar Salgado for his collaboration in reviewing the manuscript.

through extensive complementarity and proximity to the target locus. RNA Biol. 12, 136-148. doi: 10.1080/15476286.2015.1017203

Cheng, A. T., Ottemann, K. M., and Yildiz, F. H. (2015). Vibrio cholerae response regulator $\mathrm{VxrB}$ controls colonization and regulates the Type VI secretion system. PLoS Pathog. 11:e1004933. doi: 10.1371/journal.ppat.10 04933

Chiang, M. K., Lu, M. C., Liu, L. C., Lin, C. T., and Lai, Y. C. (2011). Impact of Hfq on global gene expression and virulence in Klebsiella pneumoniae. PLoS ONE 6:e22248. doi: 10.1371/journal.pone.0022248

Cressina, E., Chen, L., Moulin, M., Leeper, F. J., Abell, C., and Smith, A. G. (2011). Identification of novel ligands for thiamine pyrophosphate (TPP) riboswitches. Biochem. Soc. Trans. 39, 652-657. doi: 10.1042/BST0390652

Davies, B. W., Bogard, R. W., Young, T. S., and Mekalanos, J. J. (2012). Coordinated regulation of accessory genetic elements produces cyclic dinucleotides for V. cholerae virulence. Cell 149, 358-370. doi: 10.1016/j.cell.2012. 01.053

Davis, B. M., Quinones, M., Pratt, J., Ding, Y., and Waldor, M. K. (2005). Characterization of the small untranslated RNA RyhB and its regulon in Vibrio cholerae. J. Bacteriol. 187, 4005-4014. doi: 10.1128/JB.187.12.4005-4014. 2005

Davis, B. M., and Waldor, M. K. (2007). RNase E-dependent processing stabilizes MicX, a Vibrio cholerae sRNA. Mol. Microbiol. 65, 373-385. doi: 10.1111/j.13652958.2007.05796.X

Deng, Y., Chen, C., Zhao, Z., Zhao, J., Jacq, A., Huang, X., et al. (2016). The RNA chaperone Hfq is involved in colony morphology, nutrient utilization and oxidative and envelope stress response in Vibrio alginolyticus. PLoS ONE 11:e0163689. doi: 10.1371/journal.pone.0163689

Ding, Y., Davis, B. M., and Waldor, M. K. (2004). Hfq is essential for Vibrio cholerae virulence and downregulates ?ఠE expression. Mol. Microbiol. 53, 345-354. doi: 10.1111/j.1365-2958.2004.04142.x

Duval, M., Simonetti, A., Caldelari, I., and Marzi, S. (2014). Multiple ways to regulate translation initiation in bacteria: mechanisms, regulatory circuits, dynamics. Biochimie 114, 18-29. doi: 10.1016/j.biochi.2015. 03.007

Feliciano, J. R., Grilo, A. M., Guerreiro, S. I., Sousa, S. A., and Leitão, J. H. (2016). Hfq: a multifaceted RNA chaperone involved in virulence. Future Microbiol. 11, 137-151. doi: 10.2217/fmb.15.128

Feng, L., Rutherford, S. T., Papenfort, K., Bagert, J. D., Van Kessel, J. C., Tirrell, D. A., et al. (2015). A Qrr noncoding RNA deploys four different regulatory mechanisms to optimize quorum-sensing dynamics. Cell 160, 228-240. doi: 10.1016/j.cell.2014.11.051

Fröhlich, K. S., and Vogel, J. (2009). Activation of gene expression by small RNA. Curr. Opin. Microbiol. 12, 674-682. doi: 10.1016/j.mib.2009. 09.009 
Furukawa, K., Gu, H., Sudarsan, N., Hayakawa, Y., Hyodo, M., and Breaker, R. R. (2012). Identification of ligand analogues that control c-diGMP riboswitches. ACS Chem. Biol. 7, 1436-1443. doi: 10.1021/cb30 $0138 \mathrm{n}$

García, K., Bastías, R., Higuera, G., Torres, R., Mellado, A., Uribe, P., et al. (2013). Rise and fall of pandemic Vibrio parahaemolyticus serotype O3: K6 in southern Chile. Environ. Microbiol. 15, 527-534. doi: 10.1111/j.1462-2920.2012. 02883.x

García, K., Torres, R., Uribe, P., Hernández, C., Rioseco, M. L., Romero, J., et al. (2009). Dynamics of clinical and environmental Vibrio parahaemolyticus strains during seafood-related summer diarrhea outbreaks in southern Chile. Appl. Environ. Microbiol. 75, 7482-7487. doi: 10.1128/AEM.01662-09

Georg, J., and Hess, W. R. (2011). cis-antisense RNA, another level of gene regulation in bacteria. Microbiol. Mol. Biol. Rev. 75, 286-300. doi: 10.1128/ MMBR.00032-10

Gode-Potratz, C. J., and McCarter, L. L. (2011). Quorum sensing and silencing in Vibrio parahaemolyticus. J. Bacteriol. 193, 4224-4237. doi: 10.1128/JB.004 32-11

Hammer, B. K., and Bassler, B. L. (2007). Regulatory small RNAs circumvent the conventional quorum sensing pathway in pandemic Vibrio cholerae. Proc. Natl. Acad. Sci. U.S.A. 104, 11145-11149. doi: 10.1073/pnas.07038 60104

Hébrard, M., Kröger, C., Srikumar, S., Colgan, A., Händler, K., and Hinton, J. C. D. (2012). sRNAs and the virulence of Salmonella enterica serovar Typhimurium. RNA Biol. 9, 437-445. doi: 10.4161/rna.20480

Heidelberg, J. F., Heidelberg, K. B., and Colwell, R. R. (2002). Seasonality of chesapeake bay bacterioplankton species. Appl. Environ. Microbiol. 68, 5488-5497. doi: 10.1128/AEM.68.11.5488-5497.2002

Kery, M. B., Feldman, M., Livny, J., and Tjaden, B. (2014). TargetRNA2: identifying targets of small regulatory RNAs in bacteria. Nucleic Acids Res. 42, 124-129. doi: 10.1093/nar/gku317

Kim, T., Bak, G., Lee, J., and Kim, K.-S. (2015). Systematic analysis of the role of bacterial Hfq-interacting sRNAs in the response to antibiotics. J. Antimicrob. Chemother. 70, 1659-1668. doi: 10.1093/jac/dkv042

Kimbrough, J. H., and Stabb, E. V. (2016). Antisocial luxO mutants provide a stationary-phase survival advantage in Vibrio fischeri ES114. J. Bacteriol. 198, 673-687. doi: 10.1128/JB.00807-15

Kulesus, R. R., Diaz-Perez, K., Slechta, E. S., Eto, D. S., and Mulvey, M. A. (2008). Impact of the RNA chaperone Hfq on the fitness and virulence potential of uropathogenic Escherichia coli. Infect. Immun. 76, 3019-3026. doi: 10.1128/IAI. 00022-08

Lee, J. H., Rhee, J. E., Park, U., Ju, H. M., Lee, B. C., Kim, T. S., et al. (2007). Identification and functional analysis of Vibrio vulnificus $\mathrm{SmcR}$, a novel global regulator. J. Microbiol. Biotechnol. 17, 325-334.

Lenz, D. H., Miller, M. B., Zhu, J., Kulkarnl, R. V., and Bassler, B. L. (2005). CsrA and three redundant small RNAs regulate quorum sensing in Vibrio cholerae. Mol. Microbiol. 58, 1186-1202. doi: 10.1111/j.1365-2958.2005. 04902.x

Lenz, D. H., Mok, K. C., Lilley, B. N., Kulkarni, R. V., Wingreen, N. S., and Bassler, B. L. (2004). The small RNA chaperone Hfq and multiple small RNAs control quorum sensing in Vibrio harveyi and Vibrio cholerae. Cell 118, 69-82. doi: 10.1016/j.cell.2004.06.009

Letchumanan, V., Chan, K. G., and Lee, L. H. (2014). Vibrio parahaemolyticus: a review on the pathogenesis, prevalence, and advance molecular identification techniques. Front. Microbiol. 5:705. doi: 10.3389/fmicb.2014. 00705

Li, L., Wong, H., Nong, W., Cheung, M. K., Law, P. T. W., Kam, K. M., et al. (2014). Comparative genomic analysis of clinical and environmental strains provides insight into the pathogenicity and evolution of Vibrio parahaemolyticus. BMC Genomics 15:1135. doi: 10.1186/1471-2164-15-1135

Litwin, C. M., and Calderwood, S. B. (1994). Analysis of the complexity of gene refulation by fur in Vibrio cholerae. J. Bacteriol. 176, 240-248. doi: 10.1128/jb. 176.1.240-248.1994

Liu, J. M., Livny, J., Lawrence, M. S., Kimball, M. D., Waldor, M. K., and Camilli, A. (2009). Experimental discovery of sRNAs in Vibrio cholerae by direct cloning, 5S/ tRNA depletion and parallel sequencing. Nucleic Acids Res. 37, 1-10. doi: 10.1093/nar/gkp080
Loyola, D. E., Navarro, C., Uribe, P., García, K., Mella, C., Díaz, D., et al. (2015). Genome diversification within a clonal population of pandemic Vibrio parahaemolyticus seems to depend on the life circumstances of each individual bacteria. BMC Genomics 16:176. doi: 10.1186/s12864-015-1385-8

Lutz, C., Erken, M., Noorian, P., Sun, S., and McDougald, D. (2013). Environmental reservoirs and mechanisms of persistence of Vibrio cholerae. Front. Microbiol. 4:375. doi: 10.3389/fmicb.2013.00375

Makarova, K. S., Haft, D. H., Barrangou, R., Brouns, S. J. J., Charpentier, E., Horvath, P., et al. (2011). Evolution and classification of the CRISPR-Cas systems. Nat. Rev. Microbiol. 9, 467-477. doi: 10.1038/nrmicro2577

Malecka, E. M., Strózecka, J., Sobañska, D., and Olejniczak, M. (2015). Structure of bacterial regulatory RNAS determines their performance in competition for the chaperone protein HFQ. Biochemistry 54, 1157-1170. doi: 10.1021/bi500741d

Massé, E., Escorcia, F. E., and Gottesman, S. (2003). Coupled degradation of a small regulatory RNA and its mRNA targets in Escherichia coli. Genes Dev. 17, 2374-2383. doi: 10.1101/gad.1127103

Massé, E., and Gottesman, S. (2002). A small RNA regulates the expression of genes involved in iron metabolism in Escherichia coli. Proc. Natl. Acad. Sci. U.S.A. 99, 4620-4625. doi: 10.1073/pnas.032066599

Mey, A. R., Butz, H. A., and Payne, S. M. (2015). Vibrio cholerae csra regulates ToxR levels in response to amino acids and is essential for virulence. MBio 6, 1-11. doi: 10.1128/mBio.01064-15

Mey, A. R., Craig, S. A., and Payne, S. M. (2005a). Characterization of Vibrio cholerae RyhB: the RyhB regulon and role of ryhB in biofilm formation. Infect. Immun. 73, 5706-5719. doi: 10.1128/IAI.73.9.5706-5719.2005

Mey, A. R., Wyckoff, E. E., Kanukurthy, V., Fisher, C. R., and Payne, S. M. (2005b). Iron and Fur Regulation in Vibrio cholerae and the role of Fur in virulence. Infect Immun 73, 8167-8178. doi: 10.1128/IAI.73.12.8167

Michaux, C., Verneuil, N., Hartke, A., and Giard, J.-C. (2014). Physiological roles of small RNA molecules. Microbiology 160, 1007-1019. doi: 10.1099/mic.0. 076208-0

Nakano, M., Takahashi, A., Su, Z., Harada, N., Mawatari, K., and Nakaya, Y. (2008). Hfq regulates the expression of the thermostable direct hemolysin gene in Vibrio parahaemolyticus. BMC Microbiol. 8:155. doi: 10.1186/1471-2180-8-155

Nguyen, A., and Jacq, A. (2014). Small RNAs in the Vibrionaceae: an ocean still to be explored. Wiley Interdiscip. Rev. RNA 5, 381-392. doi: 10.1002/wrna. 1218

Oliva, G., Sahr, T., and Buchrieser, C. (2015). Small RNAs, 5' UTR elements and RNA-binding proteins in intracellular bacteria: impact on metabolism and virulence. FEMS Microbiol. Rev. 39, 331-349. doi: 10.1093/femsre/ fuv022

Oliver, J. D. (2015). The Biology of Vibrio vulnificus. Microbiol. Spectr. 3, 1-10. doi: 10.1128/microbiolspec.VE-0001-2014

Pain, A., Ott, A., Amine, H., Rochat, T., Bouloc, P., and Gautheret, D. (2015). An assessment of bacterial small RNA target prediction programs. RNA Biol. 12, 509-513. doi: 10.1080/15476286.2015.1020269

Papenfort, K., Förstner, K. U., Cong, J.-P., Sharma, C. M., and Bassler, B. L. (2015). Differential RNA-seq of Vibrio cholerae identifies the VqmR small RNA as a regulator of biofilm formation. Proc. Natl. Acad. Sci. U.S.A. 112, E766-E775. doi: $10.1073 /$ pnas.1500203112

Papenfort, K., and Vanderpool, C. K. (2015). Target activation by regulatory RNAs in bacteria. FEMS Microbiol. Rev. 39, 362-378. doi: 10.1093/femsre/fuv016

Papenfort, K., and Vogel, J. (2010). Regulatory RNA in bacterial pathogens. Cell Host Microbe 8, 116-127. doi: 10.1016/j.chom.2010.06.008

Papenfort, K., and Vogel, J. (2014). Small RNA functions in carbon metabolism and virulence of enteric pathogens. Front. Cell Infect Microbiol. 4:91. doi: 10.3389/ fcimb.2014.00091

Plagens, A., Richter, H., Charpentier, E., and Randau, L. (2015). DNA and RNA interference mechanisms by CRISPR-Cas surveillance complexes. FEMS Microbiol. Rev. 39, 442-463. doi: 10.1093/femsre/fuv019

Porcheron, G., and Dozois, C. M. (2015). Interplay between iron homeostasis and virulence: Fur and RyhB as major regulators of bacterial pathogenicity. Vet. Microbiol. 179, 2-14. doi: 10.1016/j.vetmic.2015.03.024

Porcheron, G., Habib, R., Houle, S., Caza, M., Lépine, F., Daigle, F., et al. (2014). The small RNA RyhB contributes to siderophore production and virulence of uropathogenic Escherichia coli. Infect. Immun. 82, 5056-5068. doi: 10.1128/IAI. 02287- 14 
Raghunath, P. (2015). Roles of thermostable direct hemolysin (TDH) and TDHrelated hemolysin (TRH) in Vibrio parahaemolyticus. Front. Microbiol. 5:805. doi: $10.3389 /$ fmicb.2014.00805

Rath, D., Amlinger, L., Rath, A., and Lundgren, M. (2015). The CRISPRCas immune system: biology, mechanisms and applications. Biochimie 117, 119-128. doi: 10.1016/j.biochi.2015.03.025

Repoila, F., and Darfeuille, F. (2009). Small regulatory non-coding RNAs in bacteria: physiology and mechanistic aspects. Biol. Cell 101, 117-131. doi: 10. 1042/BC20070137

Richard, A. L., Withey, J. H., Beyhan, S., Yildiz, F., and Dirita, V. J. (2010). The Vibrio cholerae virulence regulatory cascade controls glucose uptake through activation of TarA, a small regulatory RNA. Mol. Microbiol. 78, 1171-1181. doi: 10.1111/j.1365-2958.2010.07397.x

Romeo, T., Vakulskas, C. A., and Babitzke, P. (2013). Post-transcriptional regulation on a global scale: form and function of Csr/Rsm systems. Environ. Microbiol. 15, 313-324. doi: 10.1111/j.1462-2920.2012.02794.x

Rutherford, S. T., and Bassler, B. L. (2012). Bacterial quorum sensing: its role in virulence and possibilities for its control. Cold Spring Harb. Perspect. Med. 2, 1-26. doi: 10.1101/cshperspect.a012427

Rutherford, S. T., Van Kessel, J. C., Shao, Y., and Bassler, B. L. (2011). AphA and LuxR / HapR reciprocally control quorum sensing in vibrios. Genes Dev. 25, 397-408. doi: 10.1101/gad.2015011

Sabharwal, D., Song, T., Papenfort, K., and Wai, S. N. (2015). The VrrA sRNA controls a stationary phase survival factor Vrp of Vibrio cholerae. RNA Biol. 12, 186-196. doi: 10.1080/15476286.2015.1017211

Sengupta, C., Mukherjee, O., and Chowdhury, R. (2016). Adherence to intestinal cells promotes biofilm formation in Vibrio cholerae Accepted. J. Infect. Dis. 240, 1571-1578. doi: 10.1074/jbc.M115.640094

Shao, Y., and Bassler, B. L. (2012). Quorum-sensing non-coding small RNAs use unique pairing regions to differentially control mRNA targets. Mol. Microbiol. 83, 599-611. doi: 10.1111/j.1365-2958.2011.07959.x

Shao, Y., and Bassler, B. L. (2014). Quorum regulatory small RNAs repress type VI secretion in vibrio cholerae. Mol. Microbiol. 92, 921-930. doi: 10.1111/mmi. 12599

Shao, Y., Feng, L., Rutherford, S. T., Papenfort, K., and Bassler, B. L. (2013). Functional determinants of the quorum-sensing non-coding RNAs and their roles in target regulation. EMBO J. 32, 2158-2171. doi: 10.1038/emboj. 2013.155

Silveira, A. C. G., Robertson, K. L., Lin, B., Wang, Z., Vora, G. J., Vasconcelos, A. T. R., et al. (2010). Identification of non-coding RNAs in environmental vibrios. Microbiology 156, 2452-2458. doi: 10.1099/mic.0.039149-0

Smith, K. D., and Strobel, S. A. (2011). Interactions of the c-di-GMP riboswitch with its second messenger ligand. Biochem. Soc. Trans. 39, 647-651. doi: 10. 1042/BST0390647

Song, T., Sabharwal, D., Gurung, J. M., Cheng, A. T., Sjöström, A. E., Yildiz, F. H., et al. (2014). Vibrio cholerae utilizes direct sRNA regulation in expression of a biofilm matrix protein. PLOS ONE 9:e101280. doi: 10.1371/journal.pone. 0101280

Song, T., Sabharwal, D., and Wai, S. N. (2010). VrrA mediates Hfq-dependent regulation of OmpT synthesis in vibrio cholerae. J. Mol. Biol. 400, 682-688. doi: 10.1016/j.jmb.2010.05.061

Song, T., and Wai, S. N. (2009). A novel sRNA that modulates virulence and environmental fitness of Vibrio cholerae. RNA Biol. 6, 254-258. doi: 10.4161/ rna.6.3.8371

Sonnleitner, E., Hagens, S., Rosenau, F., Wilhelm, S., Habel, A., Jäger, K. E., et al. (2003). Reduced virulence of a hfq mutant of Pseudomonas aeruginosa O1. Microb. Pathog. 35, 217-228. doi: 10.1016/S0882-4010(03) 00149-9

Sorek, R., and Cossart, P. (2010). Prokaryotic transcriptomics: a new view on regulation, physiology and pathogenicity. Nat. Rev. Genet. 11, 9-16. doi: 10. $1038 / \mathrm{nrg} 2695$

Srivastava, D., and Waters, C. M. (2012). A tangled web: regulatory connections between quorum sensing and cyclic Di-GMP. J. Bacteriol. 194, 4485-4493. doi: 10.1128/JB.00379-12

Sun, H., Li, Y., Shi, X., Lin, Y., Qiu, Y., Zhang, J., et al. (2015). Association of CRISPR/Cas evolution with Vibrio parahaemolyticus virulence factors and genotypes. Foodborne Pathog. Dis. 12, 68-73. doi: 10.1089/fpd.2014. 1792
Sun, Y., Bernardy, E. E., Hammer, B. K., and Miyashiro, T. (2013). Competence and natural transformation in vibrios. Mol. Microbiol. 89, 583-595. doi: 10.1111/ mmi. 12307

Svenningsen, S. L., Waters, C. M., and Bassler, B. L. (2008). cholerae's transition out of quorum-sensing mode A negative feedback loop involving small RNAs accelerates Vibrio cholerae's transition out of quorum-sensing mode. Genes Dev. 22, 226-238. doi: 10.1101/gad.1629908

Tanabe, T., Funahashi, T., Nakao, H., Maki, J., and Yamamoto, S. (2013). The vibrio parahaemolyticus small rna ryhb promotes production of the siderophore vibrioferrin by stabilizing the polycistronic mRNA. J. Bacteriol. 195, 3692-3703. doi: 10.1128/JB.00162-13

Tanabe, T., Miyamoto, K., Tsujibo, H., Yamamoto, S., and Funahashi, T. (2015). The small RNA Spot 42 regulates the expression of the type III secretion system 1 (T3SS1) chaperone protein VP1682 in Vibrio parahaemolyticus. FEMS Microbiol. Lett. 362, 1-8. doi: 10.1093/femsle/fnv173

Teschler, J. K., Zamorano-Sánchez, D., Utada, A. S., Warner, C. J. A., Wong, G. C. L., Linington, R. G., et al. (2015). Living in the matrix: assembly and control of Vibrio cholerae biofilms. Nat. Rev. Microbiol. 13, 255-268. doi: 10. 1038/nrmicro3433

Thompson, F. L., Iida, T., and Swings, J. (2004). Biodiversity of Vibrios. Microbiol. Mol. Biol. Rev 68, 403-431. doi: 10.1128/MMBR.68.3.403-431. 2004

Tjaden, B. (2008). TargetRNA: a tool for predicting targets of small RNA action in bacteria. Nucleic Acids Res. 36, 109-113. doi: 10.1093/nar/gkn264

Tsou, A. M., Liu, Z., Cai, T., and Zhu, J. (2011). The VarS/VarA twocomponent system modulates the activity of the Vibrio cholerae quorumsensing transcriptional regulator HapR. Microbiology 157, 1620-1628. doi: 10. 1099/mic.0.046235-0

Tu, K. C., and Bassler, B. L. (2007). Multiple small RNAs act additively to integrate sensory information and control quorum sensing in Vibrio harveyi. Genes Dev. 21, 221-233. doi: 10.1101/gad.1502407

Updegrove, T. B., Shabalina, S. A., and Storz, G. (2015). How do base-pairing small RNAs evolve? FEMS Microbiol. Rev. 39, 379-391. doi: 10.1093/femsre/ fuv014

Vakulskas, C. A., Potts, A. H., Babitzke, P., Ahmer, B. M. M., and Romeo, T. (2015). Regulation of bacterial virulence by Csr (Rsm) systems. Microbiol. Mol. Biol. Rev. 79, 193-224. doi: 10.1128/MMBR.00052-14

Van Kessel, J. C., Rutherford, S. T., Cong, J.-P., Quinodoz, S., Healy, J., and Bassler, B. L. (2015). Quorum sensing regulates the osmotic stress response in Vibrio harveyi. J. Bacteriol. 197, 73-80. doi: 10.1128/JB.02246- 14

Van Kessel, J. C., Rutherford, S. T., Shao, Y., Utria, A. F., and Bassler, B. L. (2013a). Individual and combined roles of the master regulators apha and luxr in control of the Vibrio harveyi quorum-sensing regulon. J. Bacteriol. 195, 436-443. doi: 10.1128/JB.01998-12

Van Kessel, J. C., Ulrich, L. E., Zhulin, I. B., and Bassler, L. (2013b). Analysis of activator and repressor functions reveals the requirements for transcriptional control by LuxR, the master regulator of quorum sensing in Vibrio harveyi. mBio 4, e378-e313. doi: 10.1128/mBio.00378-13.Editor

Van Puyvelde, S., Vanderleyden, J., and De Keersmaecker, S. C. J. (2015). Experimental approaches to identify small RNAs and their diverse roles in bacteria - what we have learnt in one decade of MicA research. Microbiologyopen 4, 699-711. doi: 10.1002/mbo3.263

Vogel, J., and Luisi, B. F. (2011). Hfq and its constellation of RNA. Nat. Rev. Microbiol. 9, 578-589. doi: 10.1038/nrmicro2615

Wagner, E. G. H. (2013). Cycling of RNAs on Hfq. RNA Biol. 10, 619-626. doi: 10.4161/rna.24044

Wang, H., Ayala, J. C., Benitez, J. A., and Silva, A. J. (2014). The LuxR-type regulator VpsT negatively controls the transcription of rpos, encoding the general stress response regulator, in Vibrio cholerae biofilms. J. Bacteriol. 196, 1020-1030. doi: 10.1128/JB.00993-13

Waters, L. S., and Storz, G. (2009). Regulatory RNAs in Bacteria. Cell 136, 615-628. doi: 10.1016/j.cell.2009.01.043

Wen, Y., Kim, I. H., and Kim, K. S. (2016). Iron- and quorum-sensing signals converge on small quorum-regulatory RNAs for coordinated regulation of virulence factors in Vibrio vulnificus. J. Biol. Chem. 291, 14213-14230. doi: 10.1074/jbc.M116.714063

Westra, E. R., Buckling, A., and Fineran, P. C. (2014). CRISPR-Cas systems: beyond adaptive immunity. Nat. Rev. Microbiol. 12, 317-326. doi: 10.1038/nrmicro3241 
Wright, P. R., Georg, J., Mann, M., Sorescu, D. A., Richter, A. S., Lott, S., et al. (2014). CopraRNA and IntaRNA: predicting small RNA targets, networks and interaction domains. Nucleic Acids Res. 42, 119-123. doi: 10.1093/nar/gku359

Zhang, Y., Qiu, Y., Tan, Y., Guo, Z., Yang, R., and Zhou, D. (2012). Transcriptional regulation of opaR, qrr2-4 and aphA by the master quorum-sensing regulator opaR in Vibrio parahaemolyticus. PLoS ONE 7:e34622. doi: 10.1371/journal. pone. 0034622

Conflict of Interest Statement: The authors declare that the research was conducted in the absence of any commercial or financial relationships that could be construed as a potential conflict of interest.
The reviewer AOS and handling Editor declared their shared affiliation, and the handling Editor states that the process nevertheless met the standards of a fair and objective review.

Copyright $\odot 2017$ Pérez-Reytor, Plaza, Espejo, Navarrete, Bastías and Garcia. This is an open-access article distributed under the terms of the Creative Commons Attribution License (CC BY). The use, distribution or reproduction in other forums is permitted, provided the original author(s) or licensor are credited and that the original publication in this journal is cited, in accordance with accepted academic practice. No use, distribution or reproduction is permitted which does not comply with these terms. 